\title{
TSN inhibits cell proliferation, migration, invasion, and EMT through regulating miR-874/HMGB2/ $\beta$-catenin pathway in gastric cancer
}

\author{
F. YUAN ${ }^{1}$, Z. T. ZHAO ${ }^{2}$, B. JIA ${ }^{1}$, Y. P. WANG ${ }^{1}$, W. $\mathrm{LEI}^{3, *}$ \\ ${ }^{1}$ Department of Digestive Endoscopy, Affiliated Hospital of Shandong University of Traditional Chinese Medicine, Jinan, China; ${ }^{2}$ Department \\ of Special Inspection, The Second Affiliated Hospital of Shandong University of Traditional Chinese Medicine, Jinan, China; ${ }^{3}$ Chinese Medicine \\ Department, Linyi People's Hospital, Linyi, China
}

*Correspondence: taishu43ty@yeah.net

Received September 19, 2019 / Accepted January 7, 2020

\begin{abstract}
Gastric cancer (GC) is the second leading cause of cancer-associated deaths worldwide. Tanshinone IIA (TSN) is the pure extract from the root of red-rooted salvia and has been reported to inhibit the progression of GC cells. In this study, we investigated the microRNA (miRNA) mediated gene repression mechanism in TSN-administrated GC condition. The cell viability of GC was determined by 3-(4,5-dimethyl-2-thiazolyl)-2,5-diphenyl-2-H-tetrazolium bromide (MTT) assay. Cell migration and invasion were detected by transwell assays. The expression levels of epithelial-mesenchymal transition (EMT)-associated proteins ( $\mathrm{N}$-cadherin, vimentin, E-cadherin), High-mobility group box proteins 2 (HMGB2), $\beta$-catenin pathway-related proteins ( $\beta$-catenin, c-myc, cyclin D1) were detected by western blot analysis in TSN/GC. The expression patterns of miR-874 and HMGB2 in GC were determined by quantitative real-time polymerase chain reaction (qRT-PCR). The potential miR-874-targeted HMGB2 was searched via bioinformatics methods and identified by dual-luciferase reporter assays, RNA immunoprecipitation (RIP) assays, and RNA pull-down assays. Xenograft tumor model was used to evaluate biological function in vivo. TSN limited the proliferation, migration, invasion, EMT progression in GC, and these results could be inverted by the silencing of miR-874. Moreover, the putative binding sites between miR-874 and HMGB2 were predicted by starBase software online. Meanwhile, enforced expression of HMGB2, negatively correlated with that of miR-874, reversed the positive effects of TSN administration on cells. Mechanically, TSN restrained the GC progression by miR-874/ $\mathrm{HMGB} 2 / \beta$-catenin signaling in vitro. Additionally, in vivo experiments confirmed that TSN inhibited the GC progression as well. TSN restrained the GC progression by regulating miR-874/HMGB2/ $\beta$-catenin pathways in vitro and in vivo.
\end{abstract}

Key words: gastric cancer, Tanshinone IIA, proliferation, migration, EMT, miR-874/HMGB2 axis

Gastric cancer (GC) is a common malignant tumor, ranking as the second leading cause of cancer-related death around the world [1]. Despite advances in therapy during past decades, the prognosis for patients with GC is still poor, and the 5-year survival rate is less than $25 \%[2,3]$. Many researches concentrate on how to efficiently slow down the GC progression but the clinical results remain still unsatisfactory.

Many active ingredients extracted from traditional Chinese herbal medicines have proven to be candidates for cancer treatment, and more and more papers focus on the identification of novel bioactive ingredients [4-6]. Tanshinone IIA (TSN) is a major bioactive substance extracted from the root of Salvia miltiorrhiza, which has been studied in the pharmacological activities upon cardiovascular and cerebrovascular diseases $[7,8]$. Numerous studies have represented that TSN may act as a powerful supplemental ingredient in diverse kinds of cancer, such as breast cancer [9], bladder cancer [10], colorectal, and prostate cancers [11]. Although plenty of studies have shown that TSN has effective anti-tumor effects in GC $[12,13]$, the underlying molecular mechanisms remain to be explored.

MicroRNAs (miRNAs) are a variety of endogenous low molecular weight compounds with about 22 nucleotides in length. Evidence is increasingly supporting that some miRNAs exert tumor suppressor role through targeting and inhibiting the expression of multiple oncogenes. Aberrant miRNAs are widely observed in diverse types of cancers including GC [14] and are crucial in diagnosis, prognosis, and therapeutic targets in cancer $[15,16]$. Therefore, the exploration of novel functions of miRNAs is necessary for cancer therapies. Jiang et al. reported that the growth, migra- 
tion, invasion, and tumorigenicity of GC could be suppressed by the ectopic miR-874 expression [14]. Zhang et al. showed that miR-874 is downregulated in human GC tissues and cells and regulated the processes of tumor angiogenesis in vitro [17]. However, the role of miR-874 in GC has not been investigated thoroughly.

High-mobility group box proteins 2 (HMGB2) is a member of the HMGB protein family and plays a crucial role in tumor progression [18]. Recently, HMGB2 has been found to be expressed at high levels and acting as a tumor promoter in different cancers, including GC $[19,20]$. Li et al. showed that in GC cells (GCs), the HMGB2 levels were significantly reduced after the knockdown of MALAT1 and upregulated by miR-1297 inhibition [19]. Zhang et al. found that HMGB2 expression was significantly higher in multidrug-resistant GC than in parental cells, and the knockdown of HMGB2 significantly reversed multidrug resistance in GC [21]. However, the biological role and potential molecular mechanisms of HMGB2 in GC remain undefined.

The Wnt/ $\beta$-catenin signaling pathway has been implicated in a wide range of physiological and pathophysiological processes, including GC [22-24]. Nunez et al. found that the expression of Sox7 and $\beta$-catenin was obviously associated with infiltration degree, lymph node metastasis, distant metastasis, and TNM (Tumor, Lymph Node, Metastasis) staging [25]. Peng et al. confirmed that miRNA-194 promoted the progression of GC by activating Wnt/ $\beta$-catenin signaling [26].

In our study, we explored the tumor-suppressing effects of TSN on proliferation, migration, invasion, and epithelial-mesenchymal transition (EMT) progression in GC. Moreover, the regulatory mechanism of TSN in GC was further investigated in AGS and MGC-803 cells as well as xenograft models.

\section{Materials and methods}

Cell culture, administration, and transfection. Human GC cell lines (AGS and MGC-803) and human gastric mucosal cell line (GES-1) were obtained from the Shanghai Innovation Biotechnology Co., Ltd. (Shanghai, China), with $1 \%$ penicillin/streptomycin (Beyotime, Shanghai, China), cultured as previously described [27]. TSN (Sigma-Aldrich, St. Louis, MO, USA) was dissolved in dimethyl sulfoxide (DMSO, Sigma) for storage. The cells were cultured in the medium with the presence of diverse dosage of TSN $(0,2.5$, $5,10 \mu \mathrm{g} / \mathrm{ml}$ ) for $24 \mathrm{~h}, 48 \mathrm{~h}, 72 \mathrm{~h}$, and grown in the medium containing TSN at a final concentration of $10 \mu \mathrm{g} / \mathrm{ml}$ for $48 \mathrm{~h}$ [28]. miR-874 inhibitor (anti-miR-874), miR-874 mimic (miR-874), HMGB2 overexpression plasmid (HMGB2), and controls (anti-NC, miR-NC, vector) were all obtained from GenePharma (Shanghai, China). Lipofectamine 3000 (Invitrogen, Carlsbad, CA, USA) kit was used for transfection according to the manufacturer's instructions.
3-(4,5-dimethyl-2-thiazolyl)-2,5-diphenyl-2-H-tetrazolium bromide (MTT). The proliferation capacity of transfected GCs was evaluated through MTT assay. Briefly, transfected GCs $\left(2.5 \times 10^{3} /\right.$ well $)$ were seeded into 96 -well plates (Corning, Corning, NY, USA) and maintained in an incubator with $5 \% \mathrm{CO}_{2}$ at $37^{\circ} \mathrm{C}$ for $24 \mathrm{~h}, 48 \mathrm{~h}$, and $72 \mathrm{~h}$. Then, MTT $(20 \mu \mathrm{l})$ from Sigma was added to each well and kept for $4 \mathrm{~h}$. Following this, the supernatant of each well was discarded and DMSO (150 $\mu$ l, Sigma) was added for the dissolution of the formazan crystals. In the end, the Microplate Absorbance Reader (Thermo Fisher Scientific, Waltham, MA, USA) was used for the assessment of the optical density value at $450 \mathrm{~nm}$.

Transwell assay. The rate of cell migration was investigated by the transwell chamber (Corning) without Matrigel matrix (Corning), while the invasion experiment was conducted with the transwell chamber pre-coated with Matrigel matrix. The lower chamber was filled with RPMI-1640 medium with $10 \%$ FBS, while the transfected TSN-stimulated AGS and MGC-803 cells were seeded into the upper one with $100 \mu \mathrm{l}$ of serum-free medium, and the whole steps were carried out according to the manufacturer's instructions. In the end, paraformaldehyde (PFA; Sigma) was used to attach the cells located on a lower surface of the upper chamber. Cells were analyzed under a microscope after staining with crystal violet.

Western blot. RIPA buffer (Solarbio, Beijing, China) was used to isolate total proteins in cells, and proteins were quantified by a NanoDrop 3000 (Thermo Fisher Scientific). Sodium dodecyl sulfate-polyacrylamide gel electrophoresis (SDS-PAGE) was used to separate proteins, and then proteins were transferred onto polyvinylidene fluoride (PVDF) membranes. After that, the membranes were blocked in skim milk for $2 \mathrm{~h}$ at $37^{\circ} \mathrm{C}$ and then incubated with primary antibodies at $4^{\circ} \mathrm{C}$ overnight. Following $2 \mathrm{~h}$ incubation with a secondary antibody [Goat Anti-Rabbit IgG H\&L (HRP) (1:1000; ab205718, Abcam, Cambridge, $\mathrm{UK})$ ], the chemiluminescence was detected by using an ECL detection kit (Beyotime). The primary antibodies were as follows: anti-N-cadherin (anti-N-cad) (1:1000; ab76057, Abcam), anti-Vimentin (1:1000; ab137321, Abcam), antiE-cadherin (anti-E-cad) (1:1000; ab40772, Abcam), antiHMGB2 (1:1000; ab67282, Abcam), anti- $\beta$-catenin (1:1000; ab16051, Abcam), anti-c-myc (1:1000; ab32072, Abcam), anti-cyclinD1 (1:1000; ab226977, Abcam), anti-GAPDH (1:5000; ab37168, Abcam).

Quantitative real-time polymerase chain reaction (qRT-PCR). Total RNAs of cells were extracted through TRIzol reagent (Life Technologies Corporation, Carlsbad, CA, USA). Primer-Script one-step RT-PCR kit (Takara, Shiga, Japan) or miRNA Reverse Transcription kit (GeneCopoeia, FulenGen, China) was employed to synthesize the first-strand complementary DNA of miR-874 and HMGB2. The levels of miR-874 and HMGB2 were assessed via SYBR Premix Dimer Eraser Kit (Takara). The primers sequences used were presented as below: miR-874 forward (5'-TGCGGCGGCCCCACGCACCAG-3'), miR-874 reverse 
(5'-CCAGTGCAGGGTCCGAGGT-3'), HMGB2 forward (5'-GGACCCCAATGCTCCTAAAAGGCC-3'), HMGB2 reverse (5'-TGCCCTTGGCACGATATGCAGCA-3'), U6 forward (5'-GCTTCGGCAGCACATATACTAAAAT-3'), U6 reverse (5'-CGCTTCACGAATTTGCGTGTCAT-3'), GAPDH forward (5'-GACTCATGACCACAGTCCATGC-3'), GAPDH reverse (5'-AGAGGCAGGGATGATGTTCTG-3').

The expression levels of miR-874 and HMGB2 were calculated by the $2^{-\Delta \Delta \mathrm{Ct}}$ method, and glyceraldehyde-3-phosphate dehydrogenase (GAPDH) or U6 snRNA were used as an internal control for HMGB2 and miR-874.

Dual-luciferase assay. The starBase database was used for the prediction of the binding sites between miR-874 and HMGB2. Following this, the pGL3-control vector (Promega, Madison, WI, USA) with the wild type HMGB2 sequence (HMGB2 WT) and mutant HMGB2 sequence (HMGB2 MUT) (with predicted miR-874 binding sites) were constructed to verify the binding sites between miR-874 and HMGB2. Afterward, the miR-NC or miR-874 was co-transfected into GCs with luciferase reporter vectors for the dual-luciferase reporter assay, respectively. Finally, the luciferase activities of luciferase reporter vectors were evaluated through the dual-luciferase reporter assay kit (Promega).

RNA pull-down assay. RNA pull-down assay was conducted in GCs using the RNA-Protein Pull-Down Kit (Thermo Fisher Scientific) to probe the interaction between miR-874 and HMGB2. In brief, miR-NC, miR-874-WT, or miR-874-MUT were labeled with biotin and transfected into AGS and MGC-803 cells. The cell lysates were incubated for $2 \mathrm{~h}$ using the streptavidin magnetic beads. After that, the complex was eluted using the biotin elution buffer and the HMGB2 levels were assessed using the qRT-PCR assay.

RNA immunoprecipitation (RIP) assay. Magna RIP Kit (Millipore) was used for the RIP assay. Briefly, AGS and MGC-803 cells were lysed by the RIP buffer supplemented with magnetic beads and then conjugated using the antiargonaute 2 (anti-Ago2) or IgG antibodies (negative control) overnight at $4{ }^{\circ} \mathrm{C}$. After that, the protein was digested through proteinase $\mathrm{K}$ buffer, followed by the RNA purification. Finally, qRT-PCR was carried out to measure the abundances of miR-874 and HMGB2.

Xenograft tumor model. A total of $12 \mathrm{BALB} / \mathrm{c}$ male nude mice, purchased from Charles River Laboratories (Beijing, China), were randomly divided into two groups $(n=6$ per group). MGC- 803 cells $\left(2 \times 10^{6}\right)$ were injected subcutaneously into the left flank of nude mice. The tumors were formed to $100 \mathrm{~mm}^{3}$, and the mice were intraperitoneally injected with TSN $(25 \mathrm{mg} / \mathrm{kg})$ once a week for 4 times. The control group received an equal amount of saline. The tumor volume was measured using a caliper every seven days. Mice were euthanized on the 35th day under anesthesia to separate the tumor tissues for tumor weighing, qRT-PCR, and western blot assay. The experiments were approved by the Experimental Animal
Ethics Committee of the Affiliated Hospital of Shandong University of Traditional Chinese Medicine.

Statistical analysis. All data were expressed as mean \pm standard deviation (SD) and analyzed by the SPSS 17.0 software. Comparisons among different groups were analyzed using paired Student's t-test and one-way ANOVA analysis of variance. A p-value $<0.05$ was regarded as statistically significant.

\section{Results}

TSN treatment inhibited the proliferation, migration, invasion, and EMT progression in human GC in vitro. An MTT assay was performed to evaluate the inhibitory effect of TSN on GC proliferation. The results revealed that TSN significantly inhibited the proliferation of AGS cells in a dose-dependent manner (Figure 1A). Similar results were observed in MGC-803 cells (Figure 1B). After that, the migratory and invasive abilities were assayed in GCs being induced by the different dosage of TSN, the results showed that these abilities were suppressed as increased concentration of TSN (Figures 1C, 1D). Meanwhile, EMT-related proteins expressions (Vimentin, E-cad) were limited, while $\mathrm{N}$-cad was promoted by TSN administration (Figures 1E, $1 \mathrm{~F})$. A dosage of $10 \mu \mathrm{g} / \mathrm{ml}$ of TSN for $48 \mathrm{~h}$ was most effective, and so this dosage was used for subsequent experiments. These results suggest that TSN inhibited the proliferation, migration, invasion, and EMT progression in human GC.

Inhibition of miR-874 reversed the influence of TSN upon GC in vitro. To explore the role of miR-874 in GC in vitro, to begin with, we discovered the expression pattern upon miR-874 in cells, namely GES-1, AGS, and MGC-803 cells. As shown in Figure 2A, the downregulation of miR-874 could be seen in AGS and MGC-803 cells, and the levels of which was promoted by increased dosage of TSN (Figure $2 \mathrm{~B})$. The miR-874 inhibitor was used to silence the miR-874 expression in the following experiences, according to knockout efficiency in the AGS and MGC-803 cells (Figure 2C). Functional assays represented that suppressing the expression of miR-874 enhanced the cell viability in TSN/ AGS and TSN/MGC-803 cells (Figures 2D, 2E). In consistent with the tendency of MTT analysis, the deficiency of miR-874 obviously facilitated the migratory and invasive abilities on TSN-treatment AGS and MGC-803 cells (Figures 2F, 2G). Besides, (Figures 2H, 2I) the western blot assay confirmed the protein-expression was upregulated (namely $\mathrm{N}$-cad and Vimentin) or downregulated (E-cad) after cells being transfection with anti-miR-876 vector, these data performed that the EMT progression was accelerated. Taken together, deficiency of miR-874 inverted the proliferation, migration, invasion, and the development of EMT from effects on TSN-stimulation in GCs.

HMGB2 was a direct target of miR-874. To further figure out the role of miR-874 upon regulatory mechanisms in GC, the online prediction bioinformatics software starBase was 
A
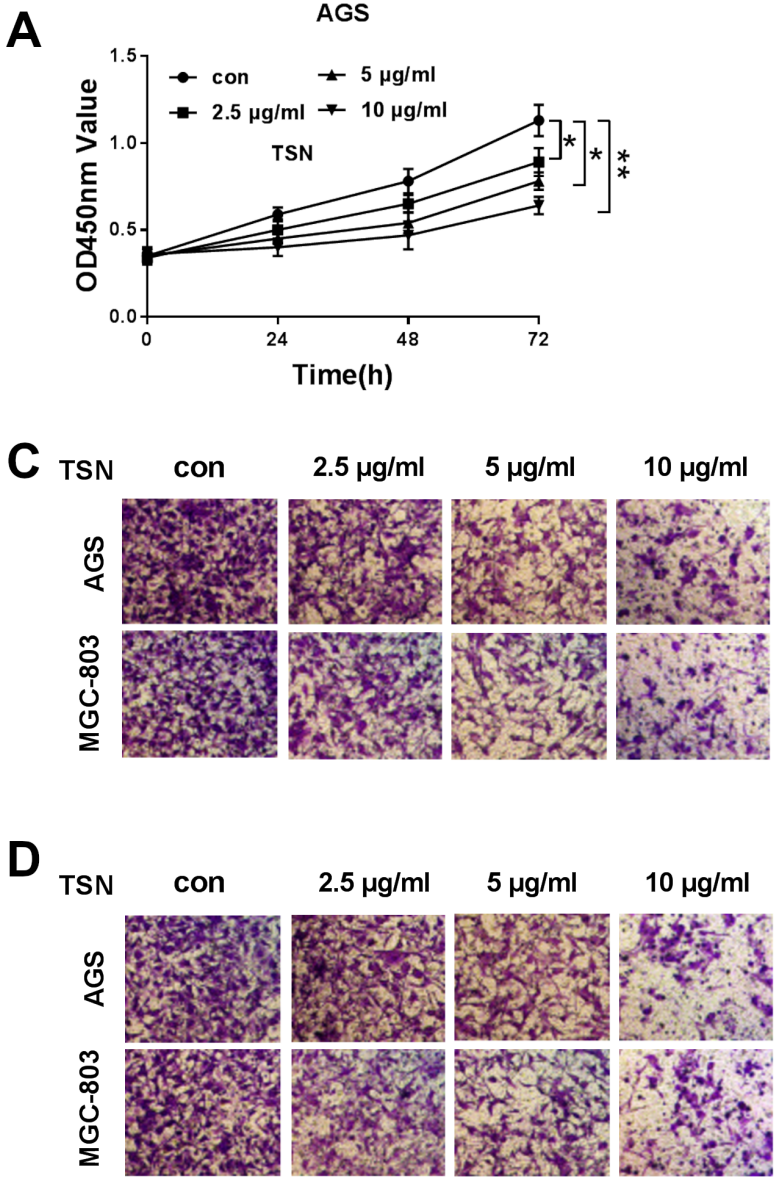

B
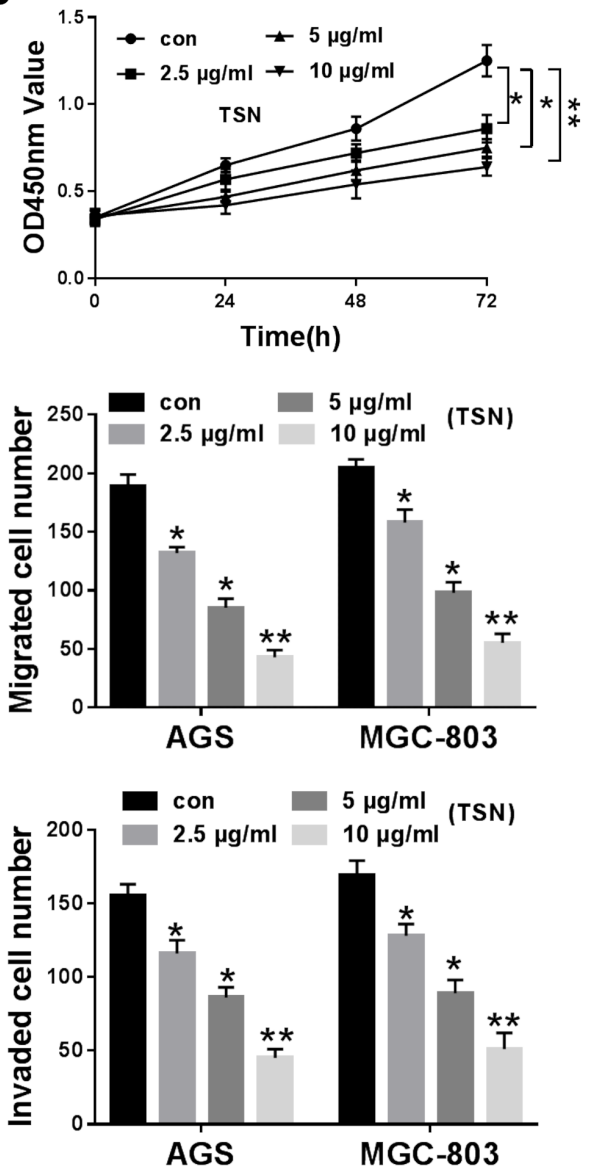

$\mathbf{E}$
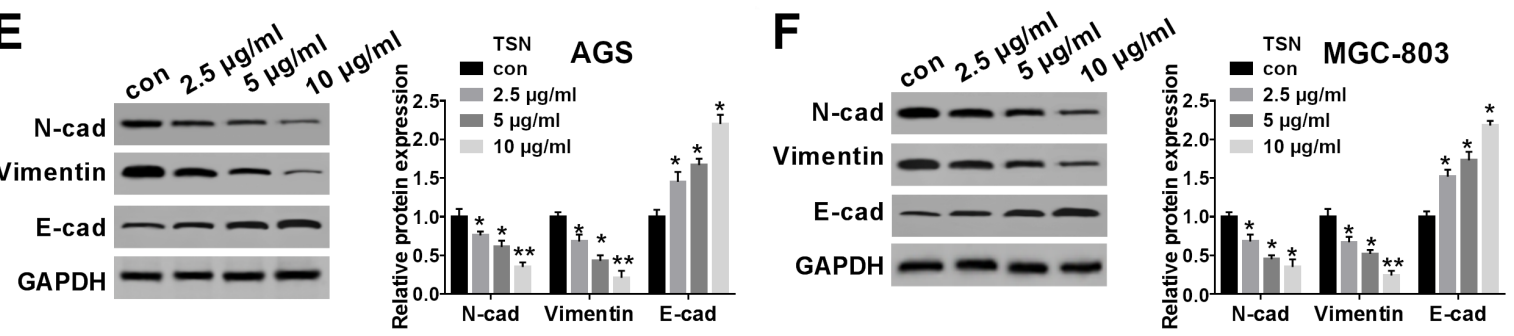

Figure 1. TSN treatment inhibited the proliferation, migration, invasion, and EMT progression in human GC in vitro. A, B) MTT assay was conducted to detect the cell viability in AGS (A) and MGC-803 cells (B) induced with different dosage $(0,2.5,5,10 \mu \mathrm{g} / \mathrm{ml}) \mathrm{of}$ TSN over the determined time spans $(24 \mathrm{~h}, 48 \mathrm{~h}, 72 \mathrm{~h}) . \mathrm{C}, \mathrm{D})$ The abilities of migration $(\mathrm{C})$ and invasion (D) were assayed by transwell assay. E, F) The EMT-associated proteins (N-cad, Vimentin, E-cad) were determined by western blot in AGS (E) and MGC-803 cells (F). ${ }^{*} \mathrm{p}<0.05,{ }^{* *} \mathrm{p}<0.01$

used to predict the potential gene binding sites. starBase v3.0 software predicted that miR-874 could target cancer-associated transcription factors, such as E2F1, E2F2, E2F7, EZH1, HMGB2, HMG20A, HMGN4, SP1, etc. We conducted the qRT-PCR assay and the detection revealed HMGB2 mRNA was obvious changed among the above-mentioned transcription factors when cells were transfected with miR-874 overexpressing plasmid. Thus, we selected HMGB2 for further experiments (Supplementary Figure S1A, S1B). We speculated that HMGB2 had a binding site with miR-874
(Figure 3A). To confirm the directed relationship between HMGB2 and miR-874, a dual-luciferase activity assay was performed and showed that co-transfection of miR-874 and HMGB2-wild type (WT) significantly decreased the luciferase activity, whereas the co-transfection of miR-NC and HMGB2-mutant (MUT) did not change the dual-luciferase activity (Figures 3B, 3C). In addition, the biotin-labeled bio-miR-874-WT group displayed a higher abundance of HMGB2 in AGS and MGC-803 cells than bio-miR874-MUT or bio-NC (Figure 3D). Meanwhile, both miR-874 

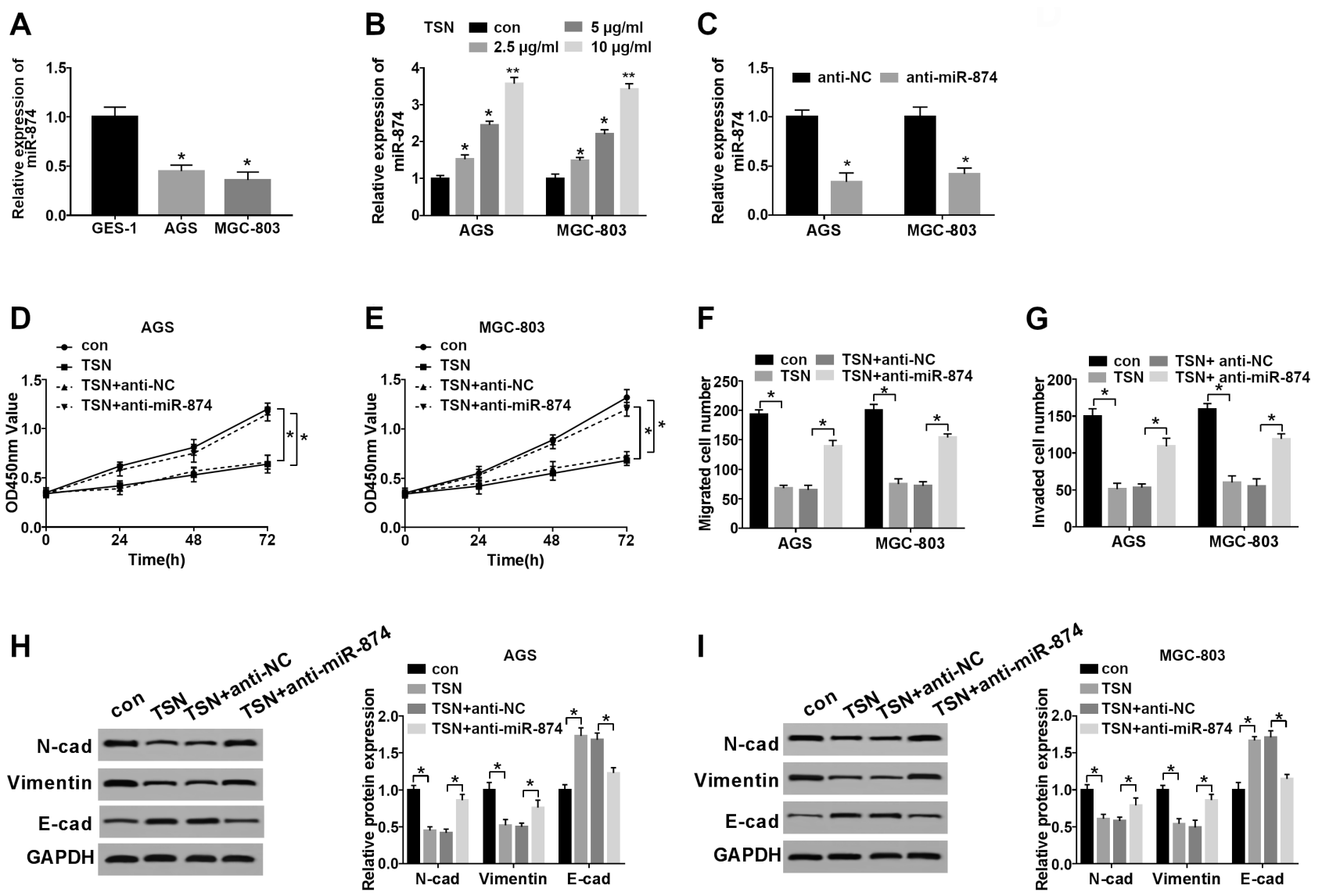

Figure 2. Inhibition of miR-874 reversed the influence of GC induced by TSN in vitro. A) The relative expression of miR-876 in cells (GES-1, AGS, and MGC-803) was analyzed by qRT-PCR. B) qRT-PCR was used to analyze the level of miR-874 in AGS and MGC-803 cells, at a determined concentration of TSN $(0,2.5,5,10 \mu \mathrm{g} / \mathrm{ml})$ for $48 \mathrm{~h}$ incubation. C) The identification of miR-874 knockdown efficiency was measured by qRT-PCR. D, E) MTT assay was performed to evaluate the viability of TSN-induced cells transfected with anti-miR-874 or anti-NC. F, G) After the vector of miR-874 transfected treatment, the abilities of migration $(\mathrm{F})$ and invasion $(\mathrm{G})$ in TSN-incubated cells were evaluated by transwell assay. $\mathrm{H}$ ) The EMT-related proteins (Ncad, Vimentin, E-cad) were determined by western blot analysis in anti-miR-874 or anti-NC cells after TSN exposure. ${ }^{\star}$ p $<0.05,{ }^{\star *} p<0.01$

and HMGB2 were significantly enriched in Ago2 immunoprecipitated pellet compared to IgG-pellet, suggesting that both miR-874 and HMGB2 were in the same RNA-induced silencing complex (Figure 3E). After that, we explored the expression of miR-874, by gain-functional experiments, and found that the level of miR-874 was upregulated (Figure 3F) with the limited expression of HMGB2 (including mRNA (Figure 3G) and protein levels (Figure 3H)) when the cells successfully transfected with miR-874 overexpression plasmid. These data above confirmed that HMGB2 was a target for miR-874, and miR-874 regulated the expression of HMGB2 in a negative manner.

TSN regulated the proliferation, migration, invasion, and EMT progression through targeting the miR-874/ HMGB2 axis in GC in vitro. To uncover the interaction between miR-874 and HMGB2, the relative expression of HMGB2 was detected in TSN/cells knocked down with miR-874 and showed that decreasing of miR-874 enhanced the expression of HMGB2 at mRNA (Figure 4A) and protein
(Figure 4B) levels, as measured by qRT-PCR analysis and western blot analysis, individually. Next, we evaluated the functional effect after TSN/cells being administrated by the HMGB2 overexpression. (Figure 4C). The identification of high expression of HMGB2 at the protein level was initially conducted by western blot. Functional assays also assured that HMGB2, exerted an oncogene, contributed to the GC progression in vitro. To the specific, enforced expression of HMGB2 restored the cell viability (Figures $4 \mathrm{D}$, 4E), migration (Figure 4F), invasion (Figure 4G), and EMT-associated proteins expression (Figures $4 \mathrm{H}, 4 \mathrm{I}$ ) due to the TSN stimulation in AGS and MGC-803 cells. These data indicated that TSN regulated the proliferation, migration, invasion, and EMT progression via targeting the miR-874/HMGB2 axis in GC in vitro.

TSN regulated the GC progression by the miR-874/ HMGB2/ $\boldsymbol{\beta}$-catenin axis in vitro. We detected levels of EMT-associated protein after gastric cancer cells transfected si- $\beta$-catenin, and data showed that its knockdown inhib- 

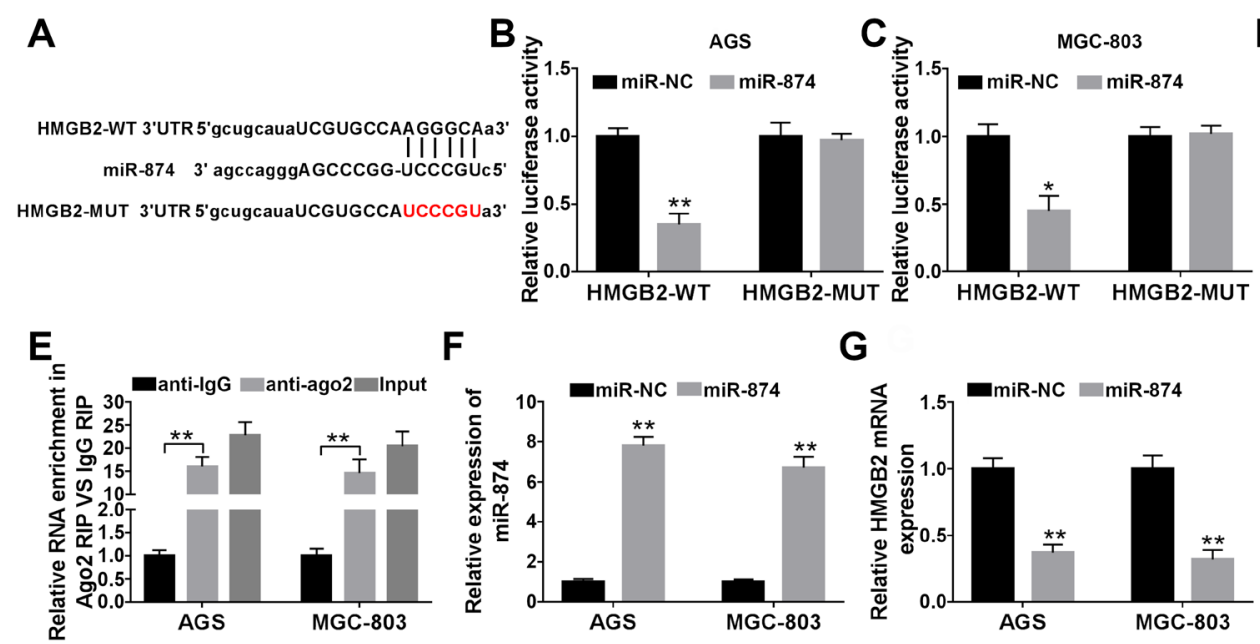

G
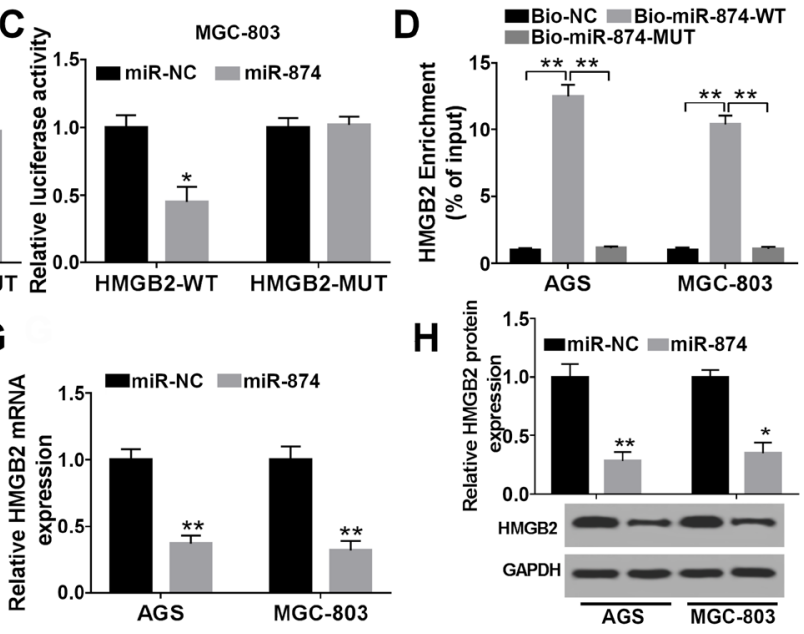

Figure 3. HMGB2 was a direct target of miR-874. A) The putative binding sites between miR-874 and HMGB2 were predicted by starBase. B, C) The predicted sites were identified by dual-luciferase reporter assay in AGS (B) and MGC-803 (C) cells. D) qRT-PCR analysis of HMGB2 enriched in AGS and MGC-803 cells transfected with Bio-miR-874-WT (WT) and Bio-miR-874-MUT (MUT) vectors in RIP assays. E) The RNA pull-down assay was performed, and the expression levels of miR-874 and HMGB2 were detected in the samples bound to the Ago2 antibody or IgG. F, G) By the construction of the gain-functional experiment, the expression of miR-874 and HMGB2 of cells was confirmed by qRT-PCR assessment. H) Western blot analysis was carried out to explore the HMGB2 and miR-874 expression in cells after miR-874 vector transfection. ${ }^{\star} \mathrm{p}<0.05,{ }^{\star *} \mathrm{p}<0.01$

A

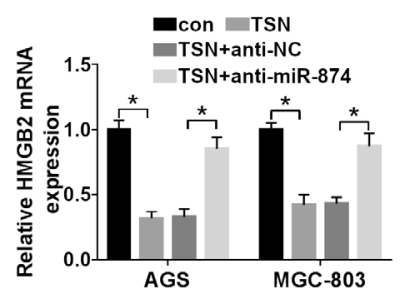

D

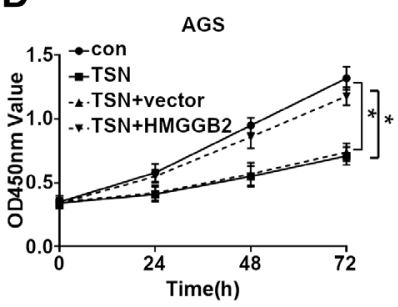

H

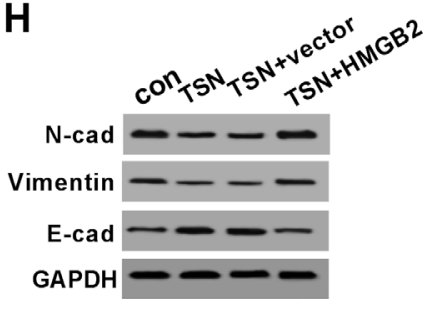

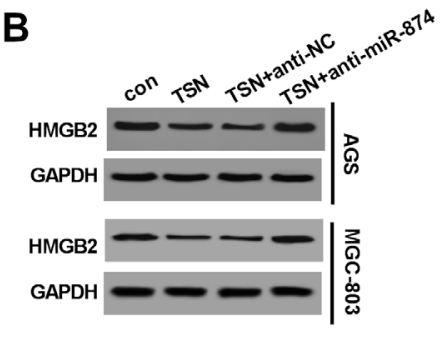

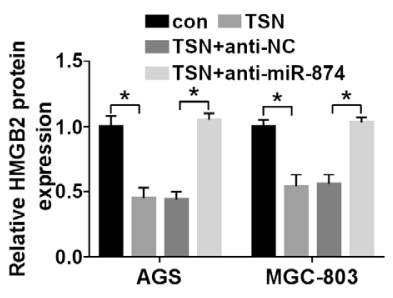

C

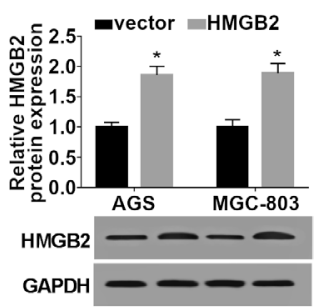

E

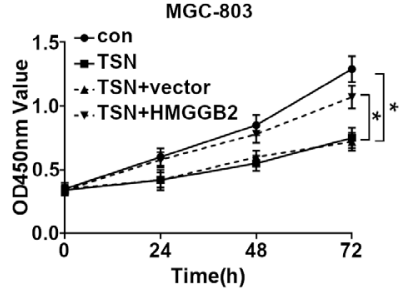

F

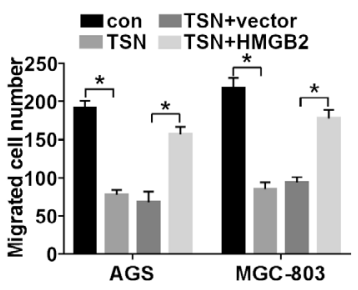

G

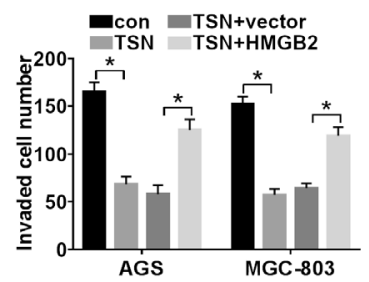

Figure 4. TSN regulated the proliferation, migration, invasion, and EMT progression through targeting the miR-874/HMGB2 signaling in GC in vitro. A, B) Anti-miR-874 vector or anti-NC was transfected into two different GC (AGS and MGC-803), the level of miR-874 of each group was confirmed by qRT-PCR (A) or western blot (B). C, D) By the overexpression vector of HMGB2 being transfected and identified by western blot (C), the cell viability was analyzed by MTT assay at stated times in TSN/cells (D). F, G) Cell migration and invasion were evaluated by transwell assay. H, I) The expressions of EMT-associated proteins (N-cad, Vimentin, E-cad) were determined by western blot assay in TSN/AGS and TSN/MGC-803 cells. ${ }^{*} \mathrm{p}<0.05$ 
ited the EMT progression (Supplementary Figure S2). The detailed regulatory effects of miR-874 on HMGB2 and the $\beta$-catenin pathway were studied in AGS and MGC-803 cells. Expressions of $\beta$-catenin, c-myc, and cyclin D1 in different groups were measured by western blot analysis (Figures $5 \mathrm{~A}$, $5 \mathrm{~B})$ after the transfection. The expression of proteins referring to the $\beta$-catenin pathway ( $\beta$-catenin, c-myc, and cyclin D1) was lower after treatment with TSN, but it could be inverted by the knockdown of miR-874 subsequently. Whereas after treatment with the overexpressed vector of HMGB2, the facilitated expression of the biomarker for the $\beta$-catenin pathway could be witnessed (Figures 5A, 5B). These results suggested miR-874/HMGB2 participated in the activation of the $\beta$-catenin pathway and regulated the $\beta$-catenin pathwayrelated proteins expression.

TSN inhibited the GC cell progression in vivo. To further examine the anti-tumor efficacy of TSN in vivo, GC cells were xenografted in nude mice. The significant differences in body weight were observed between the treatment groups (Figure 6A). Tumor volumes were significantly smaller in the TSN-treated groups $(25 \mathrm{mg} / \mathrm{kg})$ compared with the control after 35 days of treatment (Figure 6B). Excised tumor tissues exhibited the improved expression of miR-874 and reduced expression of HMGB2 (Figures 6C, 6D). In addition, TSN treatment significantly inhibited the expression of HMGB2, the biomarker of EMT (N-cad and Vimentin), meanwhile, it promoted the expression of E-cad and the activation of the $\beta$-catenin pathway (Figures $4 \mathrm{E}$ ). These results suggest that TSN inhibited the GC progression via the suppression of $\beta$-catenin signaling.

\section{Discussion}

It is reported that TSN participated in the progression of GC $[13,28,29]$. Yu et al. reported that TSN inhibited GCs (SGC-7901) proliferation and migration by downregulation of FOXM1 [13]. Su et al., in in vitro and in vivo research, found that TSN abolished the biological functions of AGS cells by impairing the protein expression of EGFR, IGFR, and arresting the PI3K/AKT/mTOR pathway [30]. Whereas, the underlying mechanisms of TSN in the development of GC have not been fully elucidated yet.

miR-874 was pervasively studied in multiple cancers, such as rhabdomyosarcoma [31], hepatocellular carcinoma [32], pancreatic adenocarcinoma [33], osteosarcomas [34], and non-small lung cancer [35]. Several studies showed that
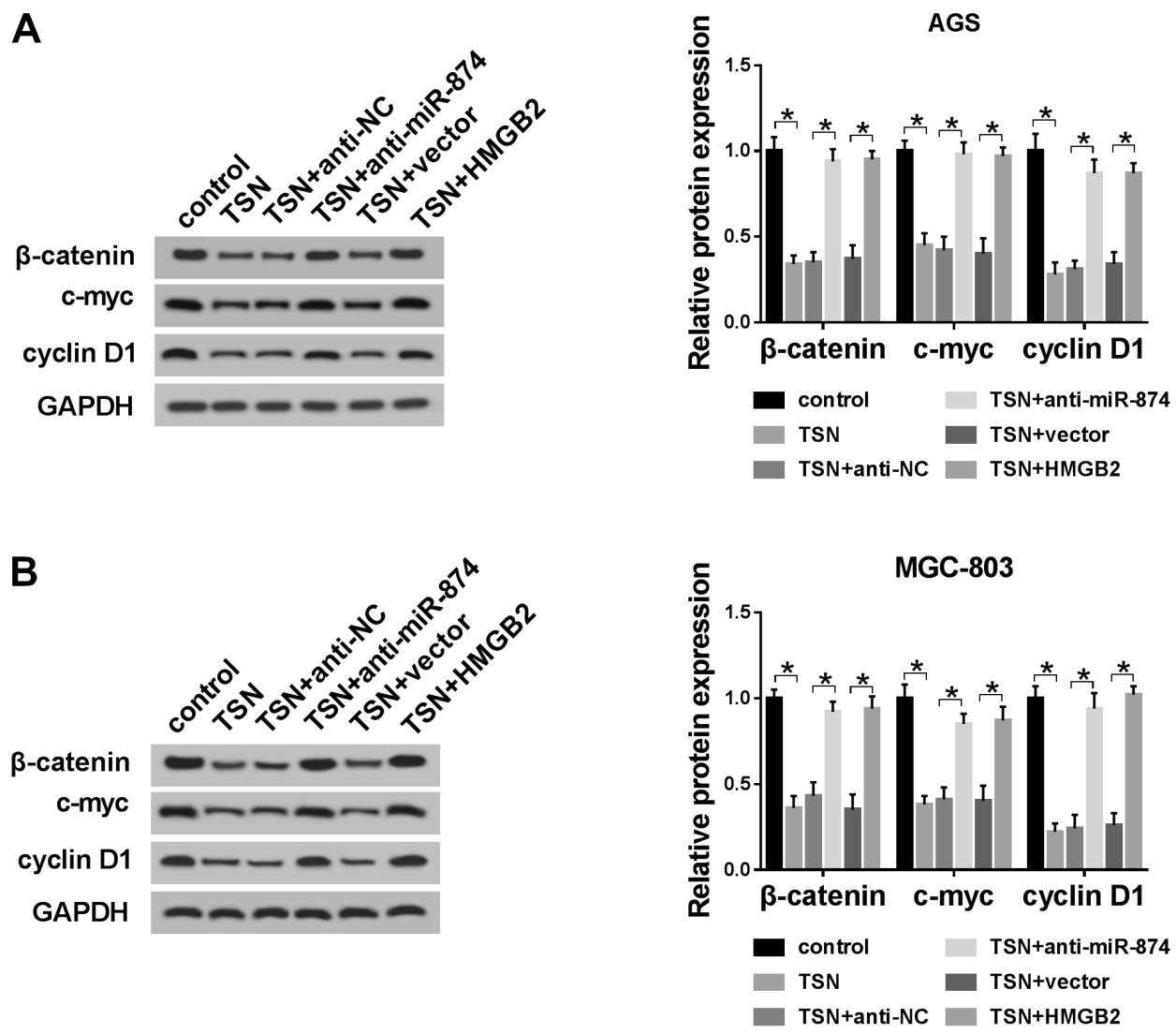

Figure 5. TSN regulated the GC progression by the miR-874/HMGB2/ $\beta$-catenin pathway in vitro. The $\beta$-catenin pathway-related proteins were explored by western blot analysis in TSN/cells when being transfected with anti-miR-874 or HMGB2 vector. A, B) The levels of $\beta$-catenin, c-myc, cyclin D1 in AGS (A), or MGC-803 (B) cells were confirmed. " $\mathrm{p}<0.05$ 
A

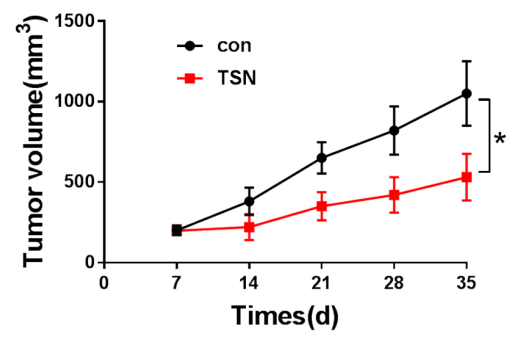

D

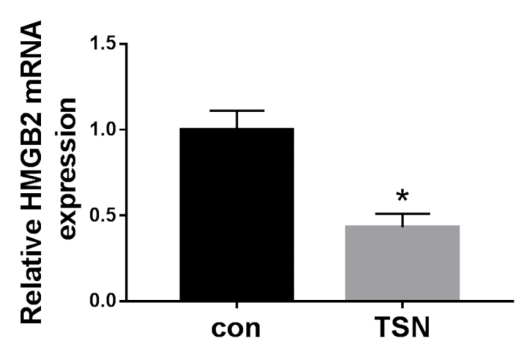

B

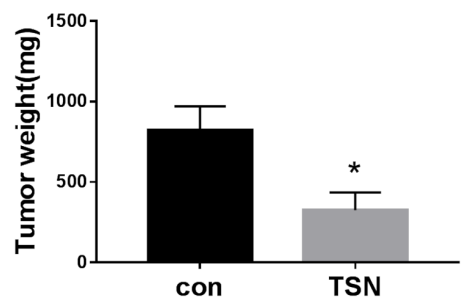

E

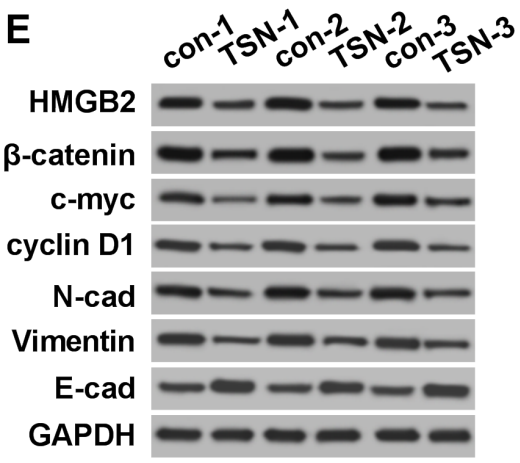

C

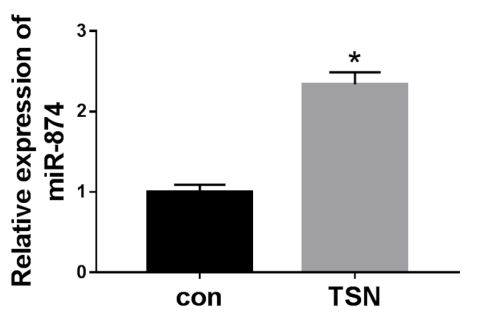

Figure 6. TSN inhibited the GC progression in vivo. Mice (6 mice/group) were subcutaneously injected with $2 \times 10^{6} \mathrm{MGC}-803$ cells or an equal volume of the vehicle into the left flank and followed by treatment with $25 \mathrm{mg} / \mathrm{kg}$ TSN after 7 days. A) Tumor volume and B) body weight were measured over time. C, D) qRT-PCR analysis was performed to measure the expression levels of miR-874 (C) and HMGB2 (D) in excised tumor tissues. E) Western blotting was performed to measure the expression of a series of biomarkers, namely HMGB2, $\beta$-catenin, c-myc, cyclin D1, N-cad, Vimentin, and E-cad, individually. ${ }^{*} \mathrm{p}<0.05$

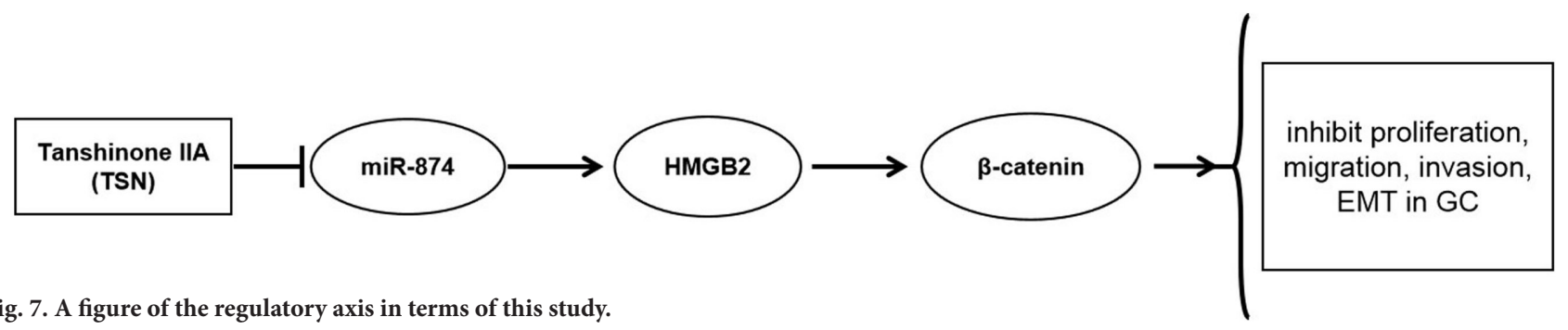

miR-874 was involved in the process of GC [36]. Huang et al. reported that miR-874 could inhibit autophagy through targeting gene autophagy-related 16-like 1 and increase the sensitivity of tumor cells to chemotherapeutic drugs [36]. A foregone study documented that upregulated levels of miR-874 limit cell growth, migration, invasion, and tumorigenicity in GC. Conversely, the opposite results could be seen in cells knocked down with miR-874 [14]. Furthermore, several pieces of evidence have demonstrated that miR-874, as a tumor inhibitor, abated angiogenesis via STAT3/VEGF-A signaling in GC [17].

Our data also showed that TSN exhibited significant tumor suppressive-effects upon proliferation, migration, invasion, and EMT progression, which was consistent with a reported paper [37-39]. Afterward, the miR-874 expression was upregulated when TSN was used to treat GCs, moreover, through loss-functional experiments, we found that the downregulated expression of miR-874 reversed the inhibitory effect of TSN on GCs. Therefore, we speculated that TSN played a role in inhibiting tumors by regulating the expression of miR-874.

$\beta$-catenin is defined as an oncogene as well as a critical part in the Wnt pathway [40]. It plays an essential role in regulating cancer cell progression, early vertebrate development, and axis formation [41]. HMGB2 was found to be involved in the activation of the $\mathrm{Wnt} / \beta$-catenin signaling pathway during GC development. A study by An et al. reported that miR-23b-3p inhibited autophagy mediated by ATG12 and HMGB2 and sensitized GC cells to chemotherapy [21]. Li et al. showed that MALAT1 functioned as a molecular sponge targeting miR-1297, antagonizing its ability to suppress HMGB2 expression [19].

To explore the regulation mechanism of miR-874 in-depth, we carried out a targeted prediction and found that 
HMGB2 might be a target gene of miR-874, which has been predicted by dual-luciferase reporter assay, confirmed by RIP assay, and RNA pull-down assay. Furthermore, the relationship between miR-874 and HMGB2 existed in a negative correlation. Subsequently, to clarify the biological function of HMGB2 in tumor cells, we performed a gain-functional experiment and data showed that the upregulated HMGB2 significantly restored the tumor-suppressing effect of TSN on proliferation, migration, invasion, and the development of EMT. We also explored the regulatory mechanism of HMGB2 on tumor promotion and found that the $\beta$-catenin pathway was activated. In detail, the biomarkers, namely $\beta$-catenin, c-myc, and cyclinD1, were upregulated in tumor cells after being HMGB2 overexpressed administration or miR-874 knocked down. Thereafter, xenograft experiments have also shown that the miR-874/HMGB2/ $\beta$-catenin signaling pathway was involved in the development of GC in vivo.

There are some limitations to this experiment. For example, this experiment did not involve clinical samples; commercial cells cannot completely simulate the situation in vivo.

In conclusion, our study uncovered that TSN worked as a tumor-inhibiting active ingredient, limited cell proliferation, migration, invasion, and EMT by targeting miR-874 in GC progression in vitro and in vivo. Additionally, this paper also revealed novel signaling of miR-874/HMGB2/ $\beta$-catenin, supplying a novel therapeutic method for GC treatment.

Supplementary information is available in the online version of the paper.

Acknowledgments: This work was supported by Jinan Science and Technology Development Plan: Clinical Study of Jianpi Disinfectant in Regulating IL-6/STAT3 Signal Pathway Intervening CAG Precancerous Lesions Based on the Theory of "Spleen Deficiency and Dynamism"(No. 2017046).

\section{References}

[1] SHEIKH IA, MIRZA Z, ALI A, ALIEV G, ASHRAF GM. A proteomics based approach for the identification of gastric cancer related markers. Curr Pharm Des 2016; 22: 804-811. https://doi.org/10.2174/1381612822666151209151848

[2] FERLAY J, SOERJOMATARAM I, DIKSHIT R, ESER S, MATHERS $C$ et al. Cancer incidence and mortality worldwide: sources, methods and major patterns in GLOBOCAN 2012. Int J Cancer 2015; 136: E359-386. https://doi. org/10.1002/ijc. 29210

[3] SIEGEL RL, MILLER KD, JEMAL A. Cancer Statistics, 2017. CA Cancer J Clin 2017; 67: 7-30. https://doi.org/10.3322/ caac. 21387

[4] WANG L, WU J, LU J, MA R, SUN D et al. Regulation of the cell cycle and PI3K/Akt/mTOR signaling pathway by tanshinone I in human breast cancer cell lines. Mol Med Rep 2015; 11: 931-939. https://doi.org/10.3892/mmr.2014.2819
[5] GUTHEIL WG, REED G, RAY A, ANANT S, DHAR A. Crocetin: an agent derived from saffron for prevention and therapy for cancer. Curr Pharm Biotechnol 2012; 13: 173-179. https://doi.org/10.2174/138920112798868566

[6] GAO H, LAMUSTA J, ZHANG WF, SALMONSEN R, LIU Y et al. Tumor Cell Selective Cytotoxicity and Apoptosis Induction by an Herbal Preparation from Brucea javanica. N Am J Med Sci (Boston) 2011; 4: 62-66. https://doi.org/10.7156/ v4i2p062

[7] WEI B, YOU MG, LING JJ, WEI LL, WANG K et al. Regulation of antioxidant system, lipids and fatty acid beta-oxidation contributes to the cardioprotective effect of sodium tanshinone IIA sulphonate in isoproterenol-induced myocardial infarction in rats. Atherosclerosis 2013; 230: 148-156. https://doi.org/10.1016/j.atherosclerosis.2013.07.005

[8] ZHOU L, ZUO Z, CHOW MS. Danshen: an overview of its chemistry, pharmacology, pharmacokinetics, and clinical use. J Clin Pharmacol 2005; 45: 1345-1359. https://doi. org/10.1177/0091270005282630

[9] LI G, SHAN C, LIU L, ZHOU T, ZHOU J et al. Tanshinone IIA inhibits HIF-1alpha and VEGF expression in breast cancer cells via mTOR/p70S6K/RPS6/4E-BP1 signaling pathway. PLoS One 2015; 10: e0117440. https://doi.org/10.1371/ journal.pone. 0117440

[10] HUANG SY, CHANG SF, LIAO KF, CHIU SC. Tanshinone IIA Inhibits Epithelial-Mesenchymal Transition in Bladder Cancer Cells via Modulation of STAT3-CCL2 Signaling. Int J Mol Sci 2017; 18: E1616. https://doi.org/10.3390/ ijms 18081616

[11] ZHANG Y, JIANG P, YE M, KIM SH, JIANG C et al. Tanshinones: sources, pharmacokinetics and anti-cancer activities. Int J Mol Sci 2012; 13: 13621-13666. https://doi.org/10.3390/ ijms 131013621

[12] SU CC. Tanshinone IIA inhibits human gastric carcinoma AGS cell growth by decreasing BiP, TCTP, Mcl1 and BclxL and increasing $\mathrm{Bax}$ and $\mathrm{CHOP}$ protein expression. Int J Mol Med 2014; 34: 1661-1668. https://doi.org/10.3892/ ijmm.2014.1949

[13] YU J, WANG X, LI Y, TANG B. Tanshinone IIA suppresses gastric cancer cell proliferation and migration by downregulation of FOXM1. Oncol Rep 2017; 37: 1394-1400. https:// doi.org/10.3892/or.2017.5408

[14] JIANG B, LI Z, ZHANG W, WANG H, ZHI X et al. miR-874 Inhibits cell proliferation, migration and invasion through targeting aquaporin-3 in gastric cancer. J Gastroenterol 2014; 49: 1011-1025. https://doi.org/10.1007/s00535-013-0851-9

[15] CROCE CM, CALIN GA. miRNAs, cancer, and stem cell division. Cell 2005; 122: 6-7. https://doi.org/10.1016/j. cell.2005.06.036

[16] HE D, WANG J, ZHANG C, SHAN B, DENG X et al. Downregulation of miR-675-5p contributes to tumor progression and development by targeting pro-tumorigenic GPR55 in non-small cell lung cancer. Mol Cancer 2015; 14: 73. https:// doi.org/10.1186/s12943-015-0342-0

[17] ZHANG X, TANG J, ZHI X, XIE K, WANG W et al. miR-874 functions as a tumor suppressor by inhibiting angiogenesis through STAT3/VEGF-A pathway in gastric cancer. Oncotarget 2015; 6: 1605-1617. https://doi.org/10.18632/oncotarget. 2748 
[18] BUSTIN M. Regulation of DNA-dependent activities by the functional motifs of the high-mobility-group chromosomal proteins. Mol Cell Biol 1999; 19: 5237-5246. https://doi. org $/ 10.1128 / \mathrm{mcb} .19 .8 .5237$

[19] LI J, GAO J, TIAN W, LI Y, ZHANG J. Long non-coding RNA MALAT1 drives gastric cancer progression by regulating HMGB2 modulating the miR-1297. Cancer Cell Int 2017; 17: 44. https://doi.org/10.1186/s12935-017-0408-8

[20] CUI G, CAI F, DING Z, GAO L. HMGB2 promotes the malignancy of human gastric cancer and indicates poor survival outcome. Hum Pathol 2019; 84: 133-141. https://doi. org/10.1016/j.humpath.2018.09.017

[21] AN Y, ZHANG Z, SHANG Y, JIANG X, DONG J et al. miR$23 \mathrm{~b}-3 \mathrm{p}$ regulates the chemoresistance of gastric cancer cells by targeting ATG12 and HMGB2. Cell Death Dis 2015; 6: e1766. https://doi.org/10.1038/cddis.2015.123

[22] DOGAN A, DEMIRCI S, APDIK H, BAYRAK OF, GULLUOGLU S et al. A new hope for obesity management: Boron inhibits adipogenesis in progenitor cells through the Wnt/beta-catenin pathway. Metabolism 2017; 69: 130-142. https://doi.org/10.1016/j.metabol.2017.01.021

[23] TAO H, YANG JJ, SHI KH, LI J. Wnt signaling pathway in cardiac fibrosis: New insights and directions. Metabolism 2016; 65: 30-40. https://doi.org/10.1016/j.metabol.2015.10.013

[24] YANG XZ, CHENG TT, HE QJ, LEI ZY, CHI J et al. LINC01133 as ceRNA inhibits gastric cancer progression by sponging miR-106a-3p to regulate APC expression and the Wnt/beta-catenin pathway. Mol Cancer 2018; 17: 126. https://doi.org/10.1186/s12943-018-0874-1

[25] NUNEZ F, BRAVO S, CRUZAT F, MONTECINO M, DE FERRARI GV. Wnt/beta-catenin signaling enhances cyclooxygenase-2 (COX2) transcriptional activity in gastric cancer cells. PLoS One 2011; 6: e18562. https://doi.org/10.1371/ journal.pone.0018562

[26] PENG Y, ZHANG X, MA Q, YAN R, QIN Y et al. MiRNA-194 activates the Wnt/beta-catenin signaling pathway in gastric cancer by targeting the negative Wnt regulator, SUFU. Cancer Lett 2017; 385: 117-127. https://doi.org/10.1016/j. canlet.2016.10.035

[27] LIU Q, LIU H, CHENG H, LI Y, LI X et al. Downregulation of long noncoding RNA TUG1 inhibits proliferation and induces apoptosis through the TUG1/miR-142/ZEB2 axis in bladder cancer cells. Onco Targets Ther 2017; 10: 2461-2471. https://doi.org/10.2147/OTT.S124595

[28] ZHANG Y, GUO S, FANG J, PENG B, ZHANG Y et al. Tanshinone IIA inhibits cell proliferation and tumor growth by downregulating STAT3 in human gastric cancer. Exp Ther Med 2018; 16: 2931-2937. https://doi.org/10.3892/ etm. 2018.6562

[29] SU CC. Tanshinone IIA inhibits gastric carcinoma AGS cells by decreasing the protein expression of VEGFR and blocking Ras/Raf/MEK/ERK pathway. Int J Mol Med 2018; 41: 2389-2396. https://doi.org/10.3892/ijmm.2018.3407
[30] SU CC, CHIU TL. Tanshinone IIA decreases the protein expression of EGFR, and IGFR blocking the PI3K/Akt/ mTOR pathway in gastric carcinoma AGS cells both in vitro and in vivo. Oncol Rep 2016; 36: 1173-1179. https://doi. org/10.3892/or.2016.4857

[31] SHANG H, LIU Y, LI Z, LIU Q, CUI W et al. MicroRNA-874 functions as a tumor suppressor in rhabdomyosarcoma by directly targeting GEFT. Am J Cancer Res 2019; 9: 668-681.

[32] ZHANG Y, WEI Y, LI X, LIANG X, WANG L et al. microRNA-874 suppresses tumor proliferation and metastasis in hepatocellular carcinoma by targeting the DOR/EGFR/ ERK pathway. Cell Death Dis 2018; 9: 130. https://doi. org/10.1038/s41419-017-0131-3

[33] HUANG WJ, WANG Y, LIU S, YANG J, GUO SX et al. Silencing circular RNA hsa_circ_0000977 suppresses pancreatic ductal adenocarcinoma progression by stimulating miR874-3p and inhibiting PLK1 expression. Cancer Lett 2018; 422: 70-80. https://doi.org/10.1016/j.canlet.2018.02.014

[34] GHOSH T, VARSHNEY A, KUMAR P, KAUR M, KUMAR $\mathrm{V}$ et al. MicroRNA-874-mediated inhibition of the major G1/S phase cyclin, CCNE1, is lost in osteosarcomas. J Biol Chem 2017; 292: 21264-21281. https://doi.org/10.1074/jbc. M117.808287

[35] PIOTTO C, BISCONTIN A, MILLINO C, MOGNATO M. Functional validation of miRNAs targeting genes of DNA double-strand break repair to radiosensitize nonsmall lung cancer cells. Biochim Biophys Acta Gene Regul Mech 2018; 1861: 1102-1118. https://doi.org/10.1016/j. bbagrm.2018.10.010

[36] HUANG H, TANG J, ZHANG L, BU Y, ZHANG X. miR-874 regulates multiple-drug resistance in gastric cancer by targeting ATG16L1. Int J Oncol 2018; 53: 2769-2779. https:// doi.org/10.3892/ijo.2018.4593

[37] ZHANG Y, WEI RX, ZHU XB, CAI L, JIN W et al. Tanshinone IIA induces apoptosis and inhibits the proliferation, migration, and invasion of the osteosarcoma MG-63 cell line in vitro. Anticancer Drugs 2012; 23: 212-219. https://doi. org/10.1097/CAD.0b013e32834e5592

[38] TANG C, XUE HL, HUANG HB, WANG XG. Tanshinone IIA inhibits constitutive STAT3 activation, suppresses proliferation, and induces apoptosis in rat C6 glioma cells. Neurosci Lett 2010; 470: 126-129. https://doi.org/10.1016/j.neulet.2009.12.069

[39] TANG H, HE H, JI H, GAO L, MAO J et al. Tanshinone IIA ameliorates bleomycin-induced pulmonary fibrosis and inhibits transforming growth factor-beta-beta-dependent epithelial to mesenchymal transition. J Surg Res 2015; 197: 167-175. https://doi.org/10.1016/j.jss.2015.02.062

[40] ARMISEN R, MARCELAIN K, SIMON F, TAPIA JC, TORO $\mathrm{J}$ et al. TRPM4 enhances cell proliferation through up-regulation of the beta-catenin signaling pathway. J Cell Physiol 2011; 226: 103-109. https://doi.org/10.1002/jcp.22310

[41] AKIVA I, BIRGUL IYISON N. MGAT1 is a novel transcriptional target of Wnt/beta-catenin signaling pathway. BMC Cancer 2018; 18: 60. https://doi.org/10.1186/s12885-0173960-7 